\title{
Factors associated with the costs of hospitalization after esophagectomy: a retrospective observational study at a three- tertiary cancer hospital in China
}

\author{
Qi Liu ${ }^{1}$, Yong-Kui Yu' ${ }^{1}$, Deng-Yun Wang ${ }^{2}$, Wen-Qun Xing ${ }^{1}$ \\ ${ }^{1}$ Department of Thoracic Surgery, Henan Cancer Hospital, The Affiliated Cancer Hospital of Zhengzhou University, Zhengzhou, China; \\ ${ }^{2}$ Department of Cardiothoracic Surgery, Huangshi Central Hospital, the Affiliated Hospital of Hubei Polytechnic University, Huangshi, China \\ Contributions: (I) Conception and design: Q Liu; (II) Administrative support: WQ Xing; (III) Provision of study materials or patients: DY Wang; \\ (IV) Collection and assembly of data: DY Wang; (V) Data analysis and interpretation: DY Wang; (VI) Manuscript writing: All authors; (VII) Final \\ approval of manuscript: All authors. \\ Correspondence to: Wen-Qun Xing. Department of Thoracic Surgery, The Affiliated Cancer Hospital of Zhengzhou University, Henan Cancer \\ Hospital, Zhengzhou 450008, China. Email: zlyyxwk2012@163.com.
}

\begin{abstract}
Background: Esophageal cancer represents a major health threat in China. Esophagectomy is the standard treatment for respectable esophageal cancer. This study aimed to investigate the costs of hospitalization in esophageal cancer patients undergoing esophagectomy, and to analyze the factors influencing these costs.

Methods: A retrospective observational study which enrolled 196 patients who underwent esophagectomy from September, 2018, to April, 2019, in the Affiliated Cancer Hospital of Zhengzhou University were conducted

Results: The median inpatient cost was $¥ 72,772$ (range, $¥ 49,796-128,771$ ). Materials accounted for $39.7 \%$ of the direct medical costs, which was the highest proportion for any of the cost components. Minimally invasive esophagectomy (MIE, OR: 0.031; 95\% CI: 0.005-0.209), cardiopathy comorbidity (OR: 0.344; 95\% CI: 0.136-0.872), and anastomotic leak (OR: 0.012; 95\% CI: 0.001-0.131) were risk factors for higher cost, while early oral feeding (OR: 3.979; 95\% CI: 1.430-11.067) was a protective factor.

Conclusions: Understanding the factors associated with high hospitalization costs will help to reduce healthcare expenditure. By controlling complications and promoting early oral feeding, the economic burden on esophagectomy patients can be relieved. Further research based on a longitudinal design is needed to investigate the full costs of hospitalization associated with esophageal cancer.
\end{abstract}

Keywords: Esophageal cancer; hospitalization cost; complication; early oral feeding

Submitted Jul 24, 2020. Accepted for publication Sep 25, 2020.

doi: $10.21037 /$ jtd-20-2770

View this article at: http://dx.doi.org/10.21037/jtd-20-2770

\section{Introduction}

Esophageal cancer (EC) poses a major threat to public health and places a heavy burden on the healthcare systems of many countries globally. In China, EC is the sixth most common cause of cancer-associated mortality; in 2015 alone, there were 24,600 new cases of EC, with an estimated 18,800 deaths (1). The demographic groups most affected by EC are middle-aged and elderly men.

The treatment of esophageal cancer should adopt the principle of individualized comprehensive treatment according to the patient's physical condition, pathological type, and invasion range to enhance the chances of curing the tumor. When surgery is feasible, esophagectomy with or without neoadjuvant or adjuvant chemotherapy is the gold standard for the treatment of EC (2). Despite the considerable advances that have been made with surgical techniques for the treatment of EC, esophagectomy is still associated with a huge economic burden for patients 
and the entire healthcare system (3). Up to $74 \%$ of patients who receive esophagectomy experience a series of complex procedures after this complex surgical procedure. Postoperative complications after esophagectomy are closely related to the prolongation of hospitalization time and long-term prognosis (4).

In China, EC is also referred to as "poor disease", due to its higher prevalence in rural regions. The costs of treating EC bring a heavy financial burden to both social resources and the patient's family.

Previous studies have primarily focused on esophagectomy techniques, nutritional programs, the extent of lymph node dissection, and patients' quality of life, with few studies having been conducted to assess the medical costs of EC treatment and their relationship with the increasing risk of EC.

This study aimed to examine the direct costs of treating EC patients who underwent esophagectomy at a tertiary cancer hospital in China, as well as the structure of these costs. Also, by regression analysis, we examined the factors influencing patients' healthcare costs, in order to gain a better understanding of the economic pressure on patients and to identify ways to reduce their financial burden.

We present the following article in accordance with the STROBE reporting checklist (available at http://dx.doi. org/10.21037/jtd-20-2770).

\section{Methods}

Data of patients' direct costs were retrieved from the hospital information system (HIS), which can record details of patients' medical expenses, clinical management, and case files.

All medical records of patients admitted to the Department of Thoracic Surgery at Henan Cancer Hospital between September, 2018, and April, 2019, were marked and identified. The exclusion criteria were as follows: (I) a lack of detailed surgical records; (II) length of stay <3 days; or (III) without tumor, node, metastases stage. After the exclusion of ineligible patients, 196 patients were included for analysis.

The study protocol was approved by the Ethics Committee of the Affiliated Cancer Hospital of Zhengzhou University (2014ys38). All procedures performed in this study involving human participants were in accordance with the Declaration of Helsinki (as revised in 2013). Individual consent for this retrospective analysis was waived.

\section{Data collection}

The medical records of eligible patients were reviewed. The main variables included: patient demographic and treatment-related characteristics; comorbidities; diagnosis; procedures; operative time and approach; length of stay (LOS); and intraoperative and postoperative complications.

The following data were collected for each patient: age; gender; smoking history; nutritional status; body mass index (BMI); American Society of Anesthesiologists (ASA) score; pathological type, differentiation grade, and tumor stage; comorbidities [e.g., diabetes, hypertension, cardiopathy, chronic obstructive pulmonary disease (COPD), or cerebrovascular disease]; treatment strategy (neoadjuvant therapy or not); surgeon experience (low, moderate, or high); esophagectomy techniques (open or minimally invasive esophagectomy); surgical case order (first, second, or third); operation time; postoperative nutritional program (early oral feeding or nil by mouth with enteral tube feeding); numbers of lymph nodes dissected; postoperative complications (i.e., cardiac, respiratory, or gastrointestinal complications, anastomotic leak, chylothorax, recurrent laryngeal nerve injury, or deep venous thrombosis); length of hospital stay (preoperative, postoperative and all); and direct medical expenses with the cost structure (material, drug, examination, treatment, surgical, and other costs). Only inpatient medical expenditure was calculated, and it was calculated in Chinese Yuan (RMB).

\section{Cost grouping}

The patients were divided into three groups according to the inpatient costs reported in their medical records: the low-cost group (66 patients, $33.7 \%$ ), the moderatecost group (65 patients, $33.2 \%$ ), and the high-cost group (65 patients, $33.2 \%)$.

\section{Statistical analysis}

Statistical analyses were performed with SPSS 21.0 statistical software. The count data were expressed as frequency and rate (\%). Pearson's $\chi^{2}$ or Fisher's exact test was used to compare the differences in inpatient characteristics between groups. The influencing factors were analyzed by ordinal logistic regression. $\mathrm{P}<0.05$ showed statistical significance. 


\section{Results}

\section{General characteristics}

The medical records of 196 patients were collected. The majority of patients were male $(73 \%)$, and $25.5 \%$ were over the age of 70 years. Of the patients, 109 (55.6\%) had a smoking history, $184(93.9 \%)$ received minimally invasive surgery, $89(45.5 \%)$ received neoadjuvant therapy, $84(42.9 \%)$ received neoadjuvant chemotherapy, and $165(84.2 \%)$ were pathologically diagnosed as squamous cell carcinoma. Preoperatively, 107 patients experienced complications including hypertension ( $\mathrm{n}=55)$, cardiopathy (including arrhythmia and coronary heart disease, $\mathrm{n}=29$ ), COPD $(n=20)$, and diabetes $(n=15)$. Eight-one patients $(41.3 \%)$ were had stage II disease, and $152(77.6 \%)$ received early oral feeding. The surgeons had high and moderate experience in $64(32.7 \%)$ and $85(43.4 \%)$ cases, respectively. Also, 125 cases (63.8\%) were the first operation of that day, and 18 cases $(9.2 \%)$ were the third operation on the same day. The patients' demographic and clinical data are shown in Table 1.

\section{Direct medical costs}

Direct medical expenditure ranged from $¥ 49,796$ to $¥ 128,771$, with a median cost of $¥ 72,772$. The results showed that material costs and drug costs accounted for $39.7 \%$ and $21.7 \%$, together accounting for more than half of the total expenditure; they were followed by treatment costs and examination fees, which accounted for $11.5 \%$ and $10.4 \%$ of the total expenditure, respectively. The median cost of operation cost was $¥ 7,480$. Table 2 shows the cost structure of the esophagectomy patients' treatment.

\section{Univariate and multivariate analysis}

Univariate analysis is shown in Table 3. No significant differences were found between age, smoking history, pathological type, degree of differentiation, tumor location, stage, operation time, case order, nutritional risk score, neoadjuvant group, hypertension, diabetes mellitus, cerebrovascular disease, or number of lymph node dissected. However, there were significant differences between the groups in gender, surgeon experience, esophagectomy techniques, postoperative nutritional program, ASA score, $\mathrm{BMI}$, preoperative cardiopathy, COPD, recurrent laryngeal nerve injury, pneumonia, anastomotic leakage, incision infection, postoperative cardiac complications, and total postoperative complications.

Based on the results of univariate analysis, ordinal logistic regression was used to analyze the influencing factors of cost in each group. As shown in Table 4, the results of multivariate regression analysis revealed that early oral feeding was a protective factor for hospitalization costs. At the same time, MIE, cardiopathy, and anastomotic leak were risk factors for high hospitalization costs. The risk of incremental inpatient costs for MIE was 3.470 times higher than that for open esophagectomy, while for patients with cardiopathy the risk was 1.067 times larger than that for patients without cardiopathy. Moreover, for patients with anastomotic leak, the cost could be 4.513 times higher than that for patients without anastomotic leak.

\section{Discussion}

Until now, esophagectomy studies have focused primarily on surgical techniques, nutritional programs, the extent of lymph node dissection, and patients' quality of life, and there is a lack of literature on inpatient costs and their influencing factors for patients with EC who undergo esophagectomy.

The results of this study show that esophagectomy techniques, postoperative nutritional program, cardiopathy, and anastomotic leak were independent risk factors.

This study analyzed the direct medical costs of EC patients who underwent esophagectomy between September 2018, and April 2019. The costs of surgical consumables and drugs ranked one and two, respectively, and together accounted for more than $61 \%$ of the total direct costs. Compared with other treatments, complex surgical procedures usually require more medical materials. Moreover, with the extensive replacement of old materials with newer and more sophisticated medical materials, the corresponding medical material costs are rising (5). Therefore, we can draw a conclusion that improving doctors' awareness of the cost of medical consumables, reducing the intermediate links of material circulation, strengthening price control, and increasing clinical rational use of consumables are important ways for medical institutions to reduce treatment costs.

Studies have reported that surgical case order and the experience of the surgeon may affect patients' hospitalization expenses (6-8). Therefore, in this study, we considered the surgical case order on the day that each operation was performed as well as the experience of the surgeon. The surgical case order was divided into first, 
Table 1 Demographic and clinical information of the patients

\begin{tabular}{|c|c|c|}
\hline Variables & $\mathrm{n}$ & $\%$ \\
\hline \multicolumn{3}{|l|}{ Gender } \\
\hline Male & 143 & 73 \\
\hline Female & 53 & 27 \\
\hline \multicolumn{3}{|l|}{ Age } \\
\hline$<70$ years & 146 & 74.5 \\
\hline$\geq 70$ years & 59 & 25.5 \\
\hline \multicolumn{3}{|l|}{ Smoking history } \\
\hline Yes & 87 & 44.4 \\
\hline No & 109 & 55.6 \\
\hline \multicolumn{3}{|l|}{ Pathological type } \\
\hline Squamous cell carcinoma & 165 & 84.2 \\
\hline Adenocarcinoma & 19 & 9.7 \\
\hline Others & 12 & 6.1 \\
\hline \multicolumn{3}{|l|}{ Differentiation } \\
\hline Poorly differentiated & 45 & 23.0 \\
\hline Moderately differentiated & 120 & 61.2 \\
\hline Well differentiated & 31 & 15.8 \\
\hline \multicolumn{3}{|l|}{ Tumor location } \\
\hline Upper & 25 & 12.8 \\
\hline Middle & 93 & 47.4 \\
\hline Lower & 78 & 39.8 \\
\hline \multicolumn{3}{|l|}{ Operation time } \\
\hline$<280 \min$ & 141 & 71.9 \\
\hline$\geq 280 \mathrm{~min}$ & 55 & 28.1 \\
\hline \multicolumn{3}{|l|}{ Neoplasm stage } \\
\hline - & 63 & 32.1 \\
\hline II & 81 & 41.3 \\
\hline III & 52 & 26.5 \\
\hline \multicolumn{3}{|l|}{ Surgeon experience } \\
\hline High & 64 & 32.7 \\
\hline Moderate & 85 & 43.4 \\
\hline Low & 47 & 24.0 \\
\hline \multicolumn{3}{|l|}{ Esophagectomy technique } \\
\hline Open & 12 & 6.1 \\
\hline MIE & 184 & 93.9 \\
\hline
\end{tabular}

Table 1 (continued)
Table 1 (continued)

\begin{tabular}{|c|c|c|}
\hline Variables & $\mathrm{n}$ & $\%$ \\
\hline \multicolumn{3}{|l|}{ Treatment strategy } \\
\hline Neoadjuvant therapy & 89 & 45.4 \\
\hline Surgery alone & 107 & 54.6 \\
\hline \multicolumn{3}{|l|}{ Surgical case order } \\
\hline $1 \mathrm{st}$ & 125 & 63.8 \\
\hline $2 n d$ & 53 & 27.0 \\
\hline $3 r d$ & 18 & 9.2 \\
\hline \multicolumn{3}{|l|}{ Nutritional status score } \\
\hline$<3$ & 188 & 95.9 \\
\hline$\geq 3$ & 8 & 4.1 \\
\hline \multicolumn{3}{|l|}{ Nutritional program } \\
\hline Early oral feeding & 152 & 77.6 \\
\hline Enteral tube feeding & 44 & 22.4 \\
\hline \multicolumn{3}{|l|}{ ASA score } \\
\hline 1 & 102 & 52.0 \\
\hline 2 & 77 & 39.3 \\
\hline 3 & 17 & 8.7 \\
\hline \multicolumn{3}{|l|}{ BMI } \\
\hline$<24$ & 99 & 50.5 \\
\hline$\geq 24$ & 97 & 49.5 \\
\hline \multicolumn{3}{|l|}{ Comorbidity } \\
\hline Yes & 107 & 54.6 \\
\hline No & 89 & 45.4 \\
\hline \multicolumn{3}{|l|}{ Hypertension } \\
\hline Yes & 55 & 28.1 \\
\hline No & 141 & 71.9 \\
\hline \multicolumn{3}{|l|}{ Diabetes } \\
\hline Yes & 15 & 7.7 \\
\hline No & 181 & 92.3 \\
\hline \multicolumn{3}{|l|}{ Cardiopathy } \\
\hline Yes & 29 & 14.8 \\
\hline No & 167 & 85.2 \\
\hline \multicolumn{3}{|l|}{ COPD } \\
\hline Yes & 20 & 10.2 \\
\hline No & 176 & 89.8 \\
\hline
\end{tabular}

Table 1 (continued) 
Table 1 (continued)

\begin{tabular}{lcc}
\hline Variables & $\mathrm{n}$ & $\%$ \\
\hline Cerebrovascular disease & & \\
Yes & 16 & 8.2 \\
No & 180 & 91.8 \\
\hline
\end{tabular}

Table 2 Cost structure

\begin{tabular}{lcc}
\hline Cost structure & Median cost $(95 \% \mathrm{Cl})$ & $\%$ \\
\hline Total & $72,772(65,758-80,943)$ & \\
Materials & $30,228(23,496-33,963)$ & 39.7 \\
Drugs & $14,410(12,548-18,187)$ & 21.7 \\
Treatment & $7,424(6,141-9,384)$ & 11.5 \\
Examination & $7,137(6,057-8,593)$ & 10.4 \\
Operation & $7,480(6,100-8,230)$ & 9.8 \\
\hline
\end{tabular}

second, and third. The patients were divided into three groups according to the experience of their surgeon: the low experience group ( $<5$ years of experience after training); the moderate experience group (5-10 years of experience); and the high experience group ( $>10$ years of experience). The effects of surgeon experience on clinical outcomes have been reported for other surgical fields, with varying results $(6,9)$. In our study, no significant difference was found in hospitalization expenses according to the operation case order or the experience of the surgeon.

MIE has developed rapidly in the past decade. The proportion of EC patients treated with MIE is increasing; of the patients in this study, $93.9 \%$ received MIE. Many studies have confirmed that MIE is associated with a lower incidence of postoperative complications, a shorter postoperative hospital stay, and higher quality of life than open surgery $(10,11)$. However, in terms of hospitalization costs, MIE does not show significant advantages. The reason for this may be that compared with open surgery, more disposable consumables are needed to perform MIE, and the consumption of consumables accounts for the highest proportion of the total expenditure. Of course, with the continuous development of instruments, the price of consumables will be further reduced. We also believe that MIE will become a protective factor for lower costs in EC treatment.

The traditional methods for providing postoperative
EC patients with nutrition include parenteral nutrition and enteral nutrition through a nasogastric tube. Our team's previous research confirmed that early oral feeding is safe and feasible for EC patients after surgery $(12,13)$. Therefore, most patients $(77.6 \%)$ in this study took oral feeding on the first day after surgery, while other patients $(22.4 \%)$ failed to complete the oral intake program postoperatively due to complications such as anastomotic leak, recurrent laryngeal nerve injury, and delayed gastric emptying. Esophageal and gastric mucosal edema observed during the operations are also the reasons that patients discontinued the oral intake program. This study showed that postoperative early oral feeding is a protective factor for low costs of EC surgery. As an important part of enhanced recovery pathways for EC, early oral feeding can bring higher quality of life for patients, which has been confirmed by many studies (13-15). The results of this study show that early oral feeding can reduce the total cost for patients, which provides a basis for the further promotion of early oral feeding.

Univariate analysis showed that preoperative comorbidity, including cardiopathy and COPD, were the influencing factors of inpatient costs, which was consistent with the findings of previous reports $(16,17)$. Patients with cardiopathy, including arrhythmia and coronary heart disease, usually require further cardiac examination before surgery. Typical additional examinations include coronary computed tomography and Holter. Patients with confirmed coronary heart disease usually need to be treated with anticoagulant therapy for 3-5 days. All of these treatments and examinations increase the patient's costs. The pulmonary function of COPD patients is also evaluated and exercised. Furthermore, oxygen therapy, atomization, and other measures are also included to enable patients to meet the requirements of surgery.

Many studies have confirmed that postoperative complications are an important factor affecting the cost of surgery (18-21). In this study, univariate analysis and multivariate analysis showed that anastomotic leakage was an independent risk factor for high costs. Meanwhile, other complications, including recurrent laryngeal nerve injury, respiratory failure, pneumonia, chylothorax, and other complications also showed significant difference in the univariate analysis. Once postoperative complications occur, the hospitalization time will be prolonged, the drug cost will be increased, and there is even an increased risk of reoperation. These factors all clearly contribute to increasing the hospitalization expenses of patients. In 
Table 3 Univariate analysis

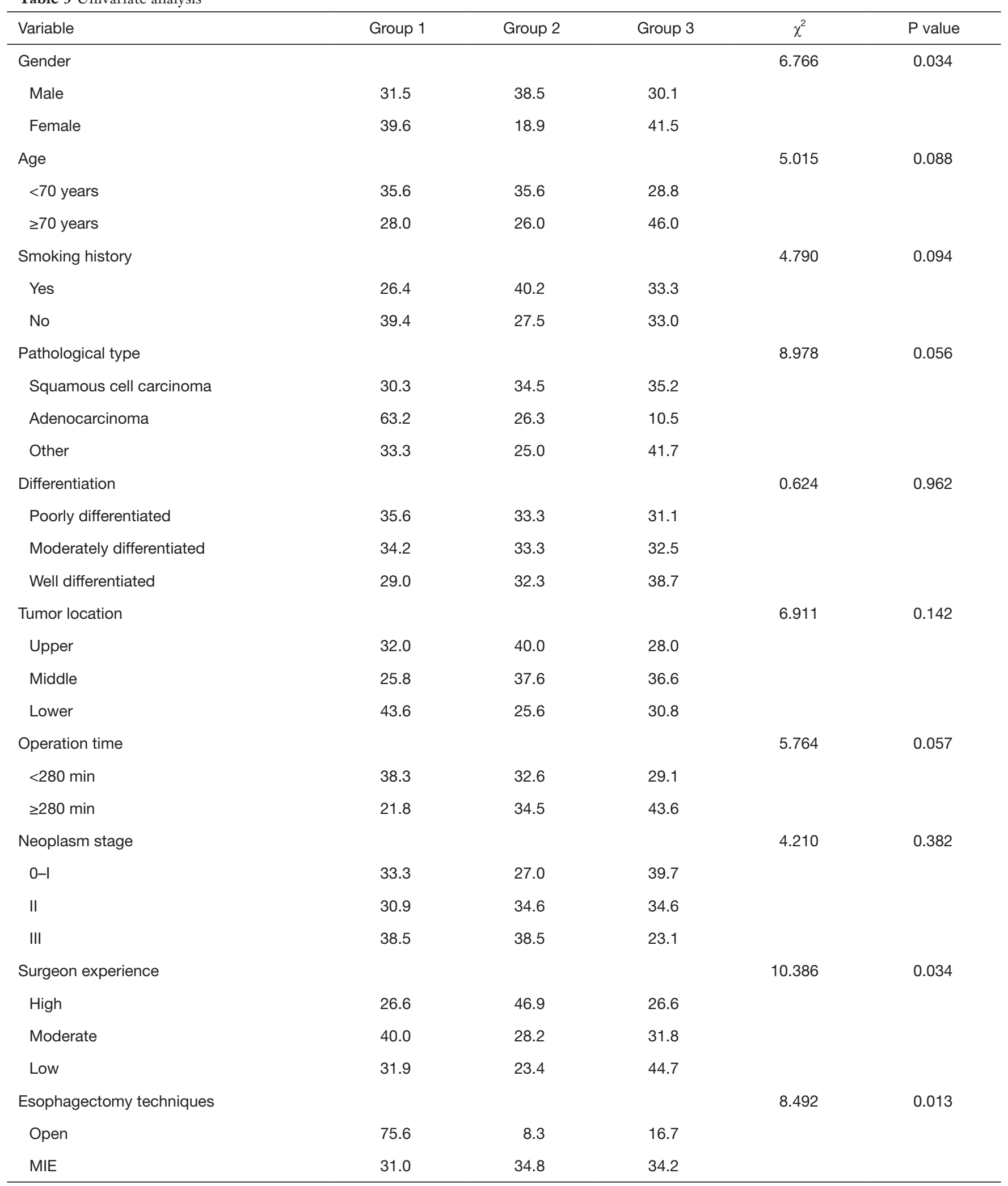

Table 3 (continued) 
Table 3 (continued)

\begin{tabular}{|c|c|c|c|c|c|}
\hline Variable & Group 1 & Group 2 & Group 3 & $\chi^{2}$ & $P$ value \\
\hline 1st & 32.8 & 35.2 & 32.0 & & \\
\hline $2 n d$ & 39.6 & 26.4 & 34.0 & & \\
\hline 3rd & 22.2 & 38.9 & 38.9 & & \\
\hline Neoadjuvant therapy & 30.3 & 40.4 & 29.2 & & \\
\hline Surgery alone & 36.4 & 27.1 & 36.4 & & \\
\hline Nutritional status score & & & & 0.415 & 0.822 \\
\hline$<3$ points & 34.0 & 33.0 & 33.0 & & \\
\hline Early oral feeding & 35.5 & 38.2 & 26.3 & & \\
\hline Enteral tube feeding & 27.3 & 15.9 & 56.8 & & \\
\hline ASA score & & & & 17.310 & 0.000 \\
\hline 1 point & 40.2 & 37.3 & 22.5 & & \\
\hline 2 points & 29.9 & 31.2 & 39.0 & & \\
\hline 3 points & 11.8 & 17.6 & 70.6 & & \\
\hline BMI & & & & 8.962 & 0.012 \\
\hline$<24 \mathrm{~kg} / \mathrm{m}^{2}$ & 39.4 & 23.2 & 37.4 & & \\
\hline Yes & 34.5 & 32.7 & 32.7 & & \\
\hline No & 33.3 & 33.3 & 33.3 & & \\
\hline Diabetes & & & & 1.331 & 0.565 \\
\hline Yes & 40.0 & 40.0 & 20.0 & & \\
\hline No & 33.1 & 32.6 & 34.3 & & \\
\hline Cardiopathy & & & & 9.952 & 0.007 \\
\hline Yes & 20.7 & 20.7 & 58.6 & & \\
\hline No & 35.9 & 35.3 & 28.7 & & \\
\hline COPD & & & & 7.237 & 0.030 \\
\hline Yes & 20.0 & 20.0 & 60.0 & & \\
\hline No & 35.2 & 34.7 & 30.1 & & \\
\hline
\end{tabular}

Table 3 (continued) 
Table 3 (continued)

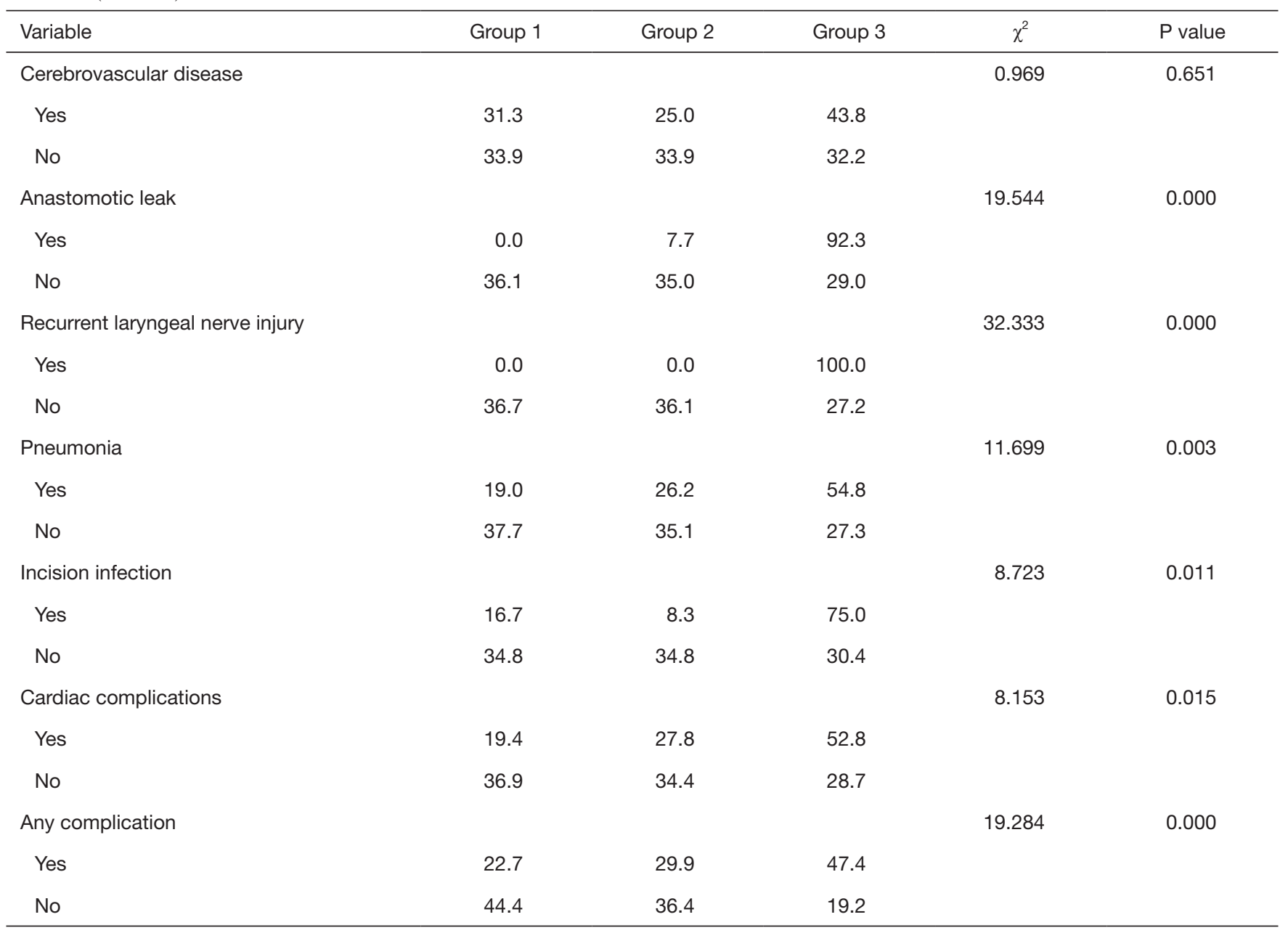

Table 4 Multivariate analysis

\begin{tabular}{lccc}
\hline Factors & Estimate & P value & OR (95\% Cl) \\
\hline Cardiopathy comorbidity & -1.067 & 0.025 & $0.344(0.136,0.872)$ \\
Anastomotic leak & -4.513 & 0.000 & $0.012(0.001,0.131)$ \\
Minimally invasive esophagectomy & -3.470 & 0.000 & $0.031(0.005,0.209)$ \\
Early oral feeding & 1.381 & 0.000 & $3.979(1.430,11.067)$ \\
\hline
\end{tabular}

accordance with the current results, previous studies have demonstrated that esophageal anastomotic leakage is associated with a significant cost burden. In our study, 13 patients had anastomotic leakage, accounting for $6.6 \%$ of the study population. The postoperative hospital stay for these 13 patients was 28 (range, 17-50) days, which was significantly higher than that of the 9 (range, 7-11) days for patients without anastomotic leakage $(\mathrm{P}<0.05)$; moreover, the costs were 99,060 (range, 86,965-111,526) vs. 72,090 (range, 65,053-79,609) for patients with and without anastomotic leakage, respectively $(\mathrm{P}<0.05)$. For patients with anastomotic leakage, the main measures that can be taken include interventional placement of a duodenal nutrition tube and fasting until the anastomotic leakage has 
healed, which will obviously increase the length of hospital stay and the costs of hospitalization. More focus should be placed on preventative measures to avoid postoperative complications, especially anastomotic leakage, at during the operation.

\section{Limitations}

This study is a single-center retrospective study, and the conclusions can only be applied to hospitals at the same level. Postoperative complications are an important factor affecting the cost of surgery; however, we did not categorize the severity of complications according to the ClavienDindo classification (CDC) system.

\section{Conclusions}

In summary, this study showed that early oral feeding, MIE, cardiopathy, and anastomotic leakage are all independent factors that affect the hospitalization cost of EC patients who undergo esophagectomy. To alleviate the economic burden on patients with esophageal cancer, efforts should be made to control complications and early oral feeding should be promoted. In future study, we will assess the influence of complications of different grades on hospitalization costs and analyze data from other centers to draw more accurate conclusions.

\section{Acknowledgments}

Funding: None.

\section{Footnote}

Reporting Checklist: The authors have completed the STROBE reporting checklist. Available at http://dx.doi. org/10.21037/jtd-20-2770

Data Sharing Statement: Available at http://dx.doi. org/10.21037/jtd-20-2770

Conflicts of Interest: All authors have completed the ICMJE uniform disclosure form (available at http://dx.doi. org/10.21037/jtd-20-2770). The authors have no conflicts of interest to declare.

Ethical Statement: The authors are accountable for all aspects of the work in ensuring that questions related to the accuracy or integrity of any part of the work are appropriately investigated and resolved. The study protocol was approved by the Ethics Committee of the Affiliated Cancer Hospital of Zhengzhou University (2014ys38). All procedures performed in this study involving human participants were in accordance with the Declaration of Helsinki (as revised in 2013). Individual consent for this retrospective analysis was waived.

Open Access Statement: This is an Open Access article distributed in accordance with the Creative Commons Attribution-NonCommercial-NoDerivs 4.0 International License (CC BY-NC-ND 4.0), which permits the noncommercial replication and distribution of the article with the strict proviso that no changes or edits are made and the original work is properly cited (including links to both the formal publication through the relevant DOI and the license). See: https://creativecommons.org/licenses/by-nc-nd/4.0/.

\section{References}

1. Bray F, Ferlay J, Soerjomataram I, et al. Global cancer statistics 2018: GLOBOCAN estimates of incidence and mortality worldwide for 36 cancers in 185 countries. CA Cancer J Clin 2018;68:394-424.

2. Drake J, Tauer K, Portnoy D, et al. Adjuvant chemotherapy is associated with improved survival in patients with nodal metastases after neoadjuvant therapy and esophagectomy. J Thorac Dis 2019;11:2546-54.

3. Giwa F, Salami A, Abioye AI. Hospital esophagectomy volume and postoperative length of stay: A systematic review and meta-analysis. Am J Surg 2018;215:155-62.

4. Guo W, Zhu L, Wu Y, et al. Endoscope-assisted mediastinal drainage therapy for anastomosis leakage after esophagectomy: a retrospective cohort study. Ann Transl Med 2019;7:747.

5. Chen Z, Leng J, Gao G, et al. Direct inpatient costs and influencing factors for patients with rectal cancer with low anterior resection: a retrospective observational study at a three-tertiary hospital in Beijing, China. BMJ Open 2018;8:e23116.

6. Boudourakis LD, Wang TS, Roman SA, et al. Evolution of the surgeon-volume, patient-outcome relationship. Ann Surg 2009;250:159-65.

7. Ellis MC, Diggs BS, Vetto JT, et al. Intraoperative oncologic staging and outcomes for lung cancer resection vary by surgeon specialty. Ann Thorac Surg 2011;92:195863; discussion 1963-4. 
8. Bagrodia A, Rachakonda V, Delafuente K, et al. Surgeon fatigue: impact of case order on perioperative parameters and patient outcomes. J Urol 2012;188:1291-6.

9. Burns EM, Faiz O. Evolution of the surgeon--volume, patient outcome relationship. Ann Surg 2010;251:991-2; author reply 992.

10. Luketich JD, Alvelo-Rivera M, Buenaventura PO, et al. Minimally invasive esophagectomy: outcomes in 222 patients. Ann Surg 2003;238:486-94; discussion 494-5.

11. Verhage RJ, Hazebroek EJ, Boone J, et al. Minimally invasive surgery compared to open procedures in esophagectomy for cancer: a systematic review of the literature. Minerva Chir 2009;64:135-46.

12. Sun HB, Liu XB, Zhang RX, et al. Early oral feeding following thoracolaparoscopic oesophagectomy for oesophageal cancer. Eur J Cardiothorac Surg 2015;47:227-33.

13. Sun HB, Li Y, Liu XB, et al. Early Oral Feeding Following McKeown Minimally Invasive Esophagectomy: An Openlabel, Randomized, Controlled, Noninferiority Trial. Ann Surg 2018;267:435-42.

14. Minig L, Biffi R, Zanagnolo V, et al. Reduction of postoperative complication rate with the use of early oral feeding in gynecologic oncologic patients undergoing a major surgery: a randomized controlled trial. Ann Surg Oncol 2009;16:3101-10.

15. Shewale JB, Correa AM, Baker CM, et al. Impact of a Fast-track Esophagectomy Protocol on Esophageal Cancer
Patient Outcomes and Hospital Charges. Ann Surg 2015;261:1114-23.

16. Murphy CC, Incalcaterra JR, Albright HW, et al. Pretreatment patient comorbidity and tobacco use increase cost and risk of postoperative complications after esophagectomy at a high-volume cancer center. J Oncol Pract 2013;9:233-9.

17. Stahl CC, Hanseman DJ, Wima K, et al. Increasing age is a predictor of short-term outcomes in esophagectomy: a propensity score adjusted analysis. J Gastrointest Surg 2014;18:1423-8.

18. Vonlanthen R, Slankamenac K, Breitenstein S, et al. The impact of complications on costs of major surgical procedures: a cost analysis of 1200 patients. Ann Surg 2011;254:907-13.

19. Short MN, Aloia TA, Ho V. The influence of complications on the costs of complex cancer surgery. Cancer 2014;120:1035-41.

20. Fu SJ, Ho VP, Ginsberg J, et al. Complications, Not Minimally Invasive Surgical Technique, Are Associated with Increased Cost after Esophagectomy. Minim Invasive Surg 2016;2016:7690632.

21. Agzarian J, Visscher SL, Knight AW, et al. The cost burden of clinically significant esophageal anastomotic leaks-a steep price to pay. J Thorac Cardiovasc Surg 2019;157:2086-92.

(English Language Editor: J. Reynolds)
Cite this article as: Liu Q, Yu YK, Wang DY, Xing WQ. Factors associated with the costs of hospitalization after esophagectomy: a retrospective observational study at a threetertiary cancer hospital in China. J Thorac Dis 2020;12(10):59705979. doi: $10.21037 /$ jtd-20-2770 